Trauma Berufskrankh 2005 · 7 [Suppl 2]: S320 DOI 10.1007/s10039-004-0948-8

Online publiziert: 5. November 2004

(c) Springer Medizin Verlag 2004
C. Knop · M. Reinhold $\cdot$ R. Schmid $\cdot$ M. Blauth

Universitätsklinik für Unfallchirurgie \& Sporttraumatologie, Medizinische Universität Innsbruck

\section{Problematik des Wirbelkörperersatzes mit Spänen und Implantaten} Kurzkommentar zum Vortrag n einem anderen Beitrag der Autoren in dieser Zeitschrift [1] zum Vortrag auf der 5. Dresdner Unfalltagung werden die aktuellen Behandlungskonzepte für Verletzungen der Brust- und Lendenwirbelsäule vorgestellt und vor dem Hintergrund des Schrifttums diskutiert. Nach dem heutigen Wissensstand sind die folgenden Fragen als offen und nicht abschließend geklärt anzuführen:

1. Welche Verletzungen in der Gruppe der Berstungsbrüche können mit mindestens gleich gutem klinischem Langzeitergebnis nichtoperativ behandelt werden?

2. Wo liegen die Grenzen der traumatischen Fehlstellung, die im Hinblick auf das Spätergebnis akzeptiert werden können?

3. Welche Methode ist am besten zur Rekonstruktion der vorderen Säule geeignet?

4. Ist durch eine technisch und röntgenologisch messbar „bessere“ ventrale $\mathrm{Re}$ konstruktion auch ein besseres klinisches Spätergebnis zu erzielen?

5. Welche Vor- und Nachteile bieten Wirbelkörperersatzimplantate für die Rekonstruktion der vorderen Säule im Vergleich zum autogenen Beckenkammspan?

6. Ist die Fusion als intervertebrale knöcherne Überbrückung bei der Behandlung mit einem Wirbelkörperersatzimplantat langfristig erforderlich?
Zur Beantwortung der Fragen können die Erfahrungen und Ergebnisse mit der kombinierten dorsoventralen Operationstechnik beitragen. Nachuntersuchungsergebnisse der ersten 50 Patienten, die mit dem Wirbelkörperersatzimplantat Synex behandelt wurden, werden in [1] präsentiert.

Als Ausblick wird die Studie „Multicenter II“ der Arbeitsgemeinschaft Wirbelsäulenchirurgie der Deutschen Gesellschaft für Unfallchirurgie vorgestellt: Im Erfassungszeitraum 2002-2003 wurden mehr als 1500 Patienten mit Verletzungen der gesamten Brust- und Lendenwirbelsäule erfasst, die einer operativen oder nicht-operativen Therapie zugeführt wurden. Mit den Nachuntersuchungsergebnissen dieses Kollektivs ist zu erwarten, dass neue Verfahren wie beispielsweise die endoskopischen und minimal-invasiven Zugangstechniken, die ventralen Techniken zur Reposition und Stabilisation, die Kyphound Vertebroplastie und auch die nichtoperativen Behandlungsmethoden in $\mathrm{Zu}$ kunft besser zu beurteilen sind.

\section{Korrespondierender Autor Univ.-Prof. Dr. C. Knop}

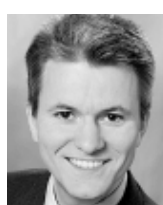

Universitätsklinik für Unfallchirurgie \& Sporttraumatologie, Medizinische Universität, Anichstraße 35, 6020 Innsbruck, Österreich E-Mail: christian.knop@uibk.ac.at
Interessenkonflikt: Der korrespondierende Autor versichert, dass keine Verbindungen mit einer Firma, deren Produkt in dem Artikel genannt ist, oder einer Firma, die ein Konkurrenzprodukt vertreibt, bestehen.

\section{Literatur}

1. Knop C, Reinhold M, Schmid R, Blauth M (2004) Fixateur interne und Wirbelkörperersatz. Indikation und Ergebnisse. Trauma Berufskrankh DOI 10.1007/s10039-004-0947-9 